\title{
Chapter C2
}

\section{THE ROLE OF ASTROCYTES, MICROGLIA, AND ENDOTHELIAL CELLS IN CORONAVIRUS- INDUCED DEMYELINATION:}

\section{Induction of cytokines and other signaling mechanisms}

\author{
Yun Li and Ehud Lavi* \\ Division of neuropathology, Department of Pathology and Laboratory Medicine, University \\ of Pennsylvania, School of Medicine, Philadelphia, PA 19104. *Present address: Department \\ of Pathology and Laboratory Medicine, Weill Medical College of Cornell University, New \\ York, NY 10021
}

Abstract: Infection of neurotropic strains of coronaviruses in susceptible animals results in acute encephalomyelitis followed by a chronic demyelinating disease, similar to multiple sclerosis (MS). Although the mechanism of chronic coronavirus-induced demyelination is not entirely clear, studies show that it is mostly immune-mediated. Astrocytes, microglia and endothelial cells play an important role in normal functions as well as in immunologic and pathologic processes in the central nervous system (CNS). The interaction between coronaviruses and these cells induces various inflammatory mediators including cytokines, chemokines, $\mathrm{MHC}$ and $\mathrm{NO}$, which acting in concert are involved in the pathogenesis of demyelination.

Key words: astrocytes, microglia, endothelial cells, coronavirus, demyelination

\section{INTRODUCTION}

Astrocytes, microglia and endothelial cells play an important role in the pathogenesis of coronavirus, mouse hepatitis virus (MHV)-induced disease in mice in several ways. All three cell-types are targets to various degrees of MHV infection during acute and chronic disease. Secondly, astrocytes and microglia are also targets of apoptotic cell death, presumably as a direct consequence of infection. In addition, astrocytes and microglia serve as reactive proliferating cells, as normally seen in viral encephalitis and other types of CNS injury. Finally all three cell-types are considered part of the CNS innate immune system, and as such are responsible for pro- 
inflammatory immune reactions. These reactions involve secretory functions of a variety of pro-inflammatory molecules including cytokines, chemokines, upregulation of major histocompatibility complex (MHC) and NO molecules and a variety of downstream signal transduction and related molecules. CNS disease, especially demyelination could be a result of a combination of viral cytopathic effect on cells and the secondary pathologic effect of toxic inflammatory mediators, thus fulfilling the concept of autoimmunity. Indirect proof for this concept comes from studies in which immuno-suppression ameliorated chronic MHV-induced demyelination. In the following paragraphs we will briefly review the normal functions of astrocytes, microglia and endothelial cells and evaluate how these functions are affected in relationship to the pathogenesis of virus-induced CNS disease.

\subsection{Astrocytes}

Historically two groups of glial cells were identified in the CNS: the macroglia, including astrocytes, oligodendrocytes, and ependymal cells, and microglia. More recently microglial cells have been identified as bone marrow derived and not of neuroectodermal origin, and therefore cannot be considered as true glial cells.

Astrocytes, the main CNS glial cells have a number of important physiological properties related to CNS homeostasis. Astrocytes have a dynamic role in regulating neuronal function (Sofroniew et al. 1999; Haydon 2000 ) by the release of neurotrophic factors, guidance of neuronal development, contributing to the metabolism of neurotransmitters, and regulating extracellular $\mathrm{pH}$ and $\mathrm{K}^{+}$levels. Astrocytes influence the formation and maintenance of the blood-brain barrier (BBB), a structure that serves to limit entry of blood-borne elements into the CNS. Astrocytic foot processes are in close apposition to the abluminal surface of the microvascular endothelium of the BBB, and soluble factors secreted by astrocytes appear to be involved in the maintenance of the BBB. Thus, astrocytes contribute to both the structural and functional integrity of the BBB (Wolburg-H 1995).

Astrocytes also serve as one of the immune effecter cells in the CNS. Astrocytes play a significant role during infectious and autoimmune diseases of the CNS. As part of the BBB, astrocytes are in close proximity to endothelial cells and secrete cytokines, chemokines and adhesion molecules (Lee et al. 2000). Cultured astrocytes express a wide range of molecules with neurotropic properties such as nervous growth factor (NGF), glialderived growth factor (GDGF), and ciliary neurotropic factor (CNTF). In response to activation by interferons astrocytes express class I and class II MHC antigens. The expression of the costimulatory molecules B7 and 
CD40 in astrocytes is controversial (Aloisi et al. 1998; Soos et al. 1999; Nguyen and Benveniste 2000).

The ability of astrocytes to function as bona fide antigen presenting cells (APCs) is also controversial. Early studies documented the ability of class II MHC-positive astrocytes to function as APCs in vitro (Fierz et al. 1985; Fontana et al. 1986; Takiguchi and Frelinger 1986). These studies clearly indicate that astrocytes exposed to IFN- $\gamma$ have the capacity to express molecules such as class II MHC, invariant chain, H2-M, B7-1, and B7-2, enabling them to efficiently process and present self antigens and activate both naive and memory $\mathrm{T}$ cells. However, there is also a large body of evidence indicating that astrocytes function as nonprofessional APCs by promoting mainly Th2 responses and/or apoptosis of T cells, which may be important for recovery from Th1-mediated CNS inflammation (Meinl et al. 1994; Weber et al. 1994).

\subsection{Microglia}

Microglia constitutes $12 \%$ of the cells in the CNS, which are the principal immune cells in the CNS and play a significant role in the host defense against invading microorganisms. The CNS endogenous microglia share many properties with macrophages, having developed from a common precursor cells (Nelson et al. 2002). Features common to microglia and systemic macrophages include the expression of innate immune receptors and the ability to phagocytose pathogens, cells or cellular debris (Ling and Wong 1993; Williams et al. 1994).

The functions of microglia in the CNS include phagocytosis, antigen presentation, production and release of cytokines, eicosanoids, complement components, and excitatory amino acids such as glutamate, oxidative radicals, and nitric oxide (Gehrmann et al. 1995). Three states of microglia have been identified based on developmental and pathophysiologic studies: (1) the resting, ramified microglia present in normal CNS; (2) the activated non-phagocytic microglia found in areas involved in CNS inflammation (3) the reactive, phagocytic microglia observed in areas of trauma and infection. Microglia can express class I and II MHC antigens, Fc receptors (I-III), complement receptors (CR1, CR2, CR4), $\beta 2$-integrins, intercellular adhesion molecule-1 (ICAM-1), and costimulatory molecules B7-1 and B7-2 (Minagar et al. 2002). Numerous studies have confirmed the role of microglia as important APCs within the CNS (Aloisi et al. 2000; Becher et al. 2000), while the role of astrocytes remains controversial.

\subsection{Endothelial cells}

Traditionally, the CNS has been regarded as an immunologically privileged site. The CNS lacks lymphatic vessels and is sealed by the BBB. 
The cellular basis for the BBB is at the levels of both the CNS microvascular endothelial cells and the choroid plexus epithelial cells. The BBB is an important mechanism for protecting the brain from fluctuations in plasma composition and from circulating agents such as neurotransmitters and xenobiotics capable of disturbing neural function (Abbott and Romero 1996). The barrier also plays an important role in the homeostatic regulation of the brain microenvironment necessary for the stable and coordinated activity of neurons (Abbott 2002). The brain endothelium has lower levels of endocytosis/transcytosis than peripheral capillaries, but has a number of specific transport and enzyme systems, which regulate molecular traffic across the endothelial cells. The brain endothelium also contains specific enzymatic systems such as monoamine oxidase that support the protective and detoxifying roles of the BBB. Cerebral microvascular endothelial cells are also considered as potential APCs because of their large cumulative surface and their unique anatomical location between circulating $T$ cells and the extra-vascular sites of antigen exposure. Thus BBB covers a number of static and dynamic properties that enable the endothelium to protect and regulate the brain microenvironment (Abbott and Romero 1996).

The BBB phenotype develops under the influence of associated brain cells, especially astrocytes. In vitro cell culture models have provided a great deal of information about the key role of astrocytes in the induction of the BBB phenotype in brain endothelium (Bauer and Bauer 2000). The chemical nature of the glial-produced inductive signal(s) is currently unclear. TGF- $\beta$ (Tran et al. 1999), GDNF (Utsumi et al. 2000), bFGF (Sobue et al. 1999), IL-6 and hydrocortisone (Hoheisel et al. 1998) have been shown to modulate endothelial differentiation and induction. In addition to a role in barrier induction and maintenance, astrocytes may play active roles in modulating $\mathrm{BBB}$ permeability by the release of several humoral agents including glutamate, aspartate, taurine, ATP, ET-1, NO, TNF $\alpha$, MIP2 and $\mathrm{IL}-1 \beta$, although the regulation of this release is not well understood (Abbott 2002). Interestingly, it has recently been shown that endothelial cells induce the differentiation of astrocyte precursor cells into astrocytes in vitro, which is mediated by endothelial cell production of leukemia inhibitory factor (LIF) (Mi et al. 2001). Thus endothelium and astrocytes are involved in two-way induction. It is clear that endothelial cells are involved in both long- and short-term chemical communication with neighboring cells, with astrocytes being of particular importance.

Maintenance of the adult BBB appears to depend on continuing exchange of inductive signals between glia and endothelium, and disturbance of this induction may be instrumental in several neuropathologies involving BBB dysfunction, such as tumors and MS. 


\section{Animal models of coronavirus induced demyelination}

Coronaviruses constitute a large group of positive-stranded RNA viruses that are associated with a wide variety of respiratory, gastrointestinal, and neurological diseases in animals and humans. However there is no conclusive evidence that any human neurological diseases, such as MS, may occur as a result of human coronavirus infection.

MS is an inflammatory demyelinating disease of the CNS that is characterized by mononuclear cell infiltration into the CNS, myelin degradation and oligodendrocyte loss. It is the most common neurological disease affecting young adults (Ewing and Bernard 1998). The etiology and pathogenesis of MS have yet to be elucidated but are probably multifactorial, involving both genetic and environmental factors (Ewing and Bernard 1998). Several viruses have been associated with demyelinating processes including human coronaviruses ( $\mathrm{HCOV}$ ) and murine coronavirus (MHV). Intracerebral infection of susceptible mice with neuroadapted strains of mouse hepatitis virus (MHV) results in an acute encephalomyelitis followed by a chronic demyelinating disease similar to the pathology of the human MS (Lavi et al. 1984; Lavi et al. 1986; Houtman and Fleming 1996; Lane and Buchmeier 1997), serving as models for this diseases. The two strains of MHV that have been used in the majority of studies of MHV infection in the CNS are MHV-A59 and JHM (MHV-4).

The mechanism of MHV induced demyelination is still not well understood. Although it was initially believed to result from direct viral lysis of oligodendrocytes, chronic demyelination was demonstrated to be largely immune mediated in more recent reports. The immune response to MHV infection is critical in host defense as well as the development of demyelination (Houtman and Fleming 1996; Wu et al. 2000). However, the precise role of individual components of the immune system in this process is not known and need intense investigation.

\section{The role of astrocytes, microglia, and endothelial cells in MHV-induced CNS demyelination}

The fact that the nude mice were unable to clear virus and still develop demyelination suggests that conventionally educated $\mathrm{T}$ cells are not an essential component for demyelination. That means that either the $\gamma \delta$ subset of $\mathrm{T}$ cells, natural killer cells, or microglia, astrocytes, or even endothelial cells, may participate and/or be responsible for the demyelinating process.

\subsection{The innate immune system of the CNS}

To protect the host from the invasion of foreign organisms and pathogenic insults, the immune system has evolved into two parts: one is 
responsible for immediate, relatively generic, action against external agents, known as the "the innate immune system". The other component of the immune system responds specifically to an external threat and requires plasticity and memory, which is called the "adaptive or acquired immune system". These two systems are not separate, but are functionally intertwined and the actions of one have a profound effect on the other (Medzhitov and Janeway 1997).

$\mathrm{B}$ and $\mathrm{T}$ lymphocytes are the cellular members of the adaptive immune system. These cells are generated in primary lymphoid tissues (bone marrow, thymus) and re-circulated in secondary lymphoid structures (lymphnode, spleen). The innate immune system on the other hand uses mainly phagocytic cells (including monocytes/macrophages, and polymorphonuclear phagocytes) as a first line of defense against foreign structures. Astrocytes and microglia are the principal innate immune cells in the CNS concomitant with inflammatory brain disease and play a significant role in the host defense against invading microorganisms.

\subsection{The immune response within the CNS upon viral infection}

Although the CNS has been characterized as an immune privileged site, it is also the site of blood-borne inflammation, either in response to exogenous antigens (infection) or as a result of disrupted peripheral tolerance to self antigens (autoimmnunity). The microenvironment of the CNS, shaped by its structural and cellular components, participates in the physiological immune process that occurs within the CNS and is at least in part responsible for the extent of immune response within the CNS upon viral infections. Glial and microglial cells play a pivotal role in both acute and chronic phases of coronavirus infection, which could lead to the production of a variety of inflammatory molecules in glial cells including cytokines, chemokines, MHC and nitric oxide (NO) that have been associated with the pathogenesis of demyelination.

\subsection{MHC class I and II expression}

The MHC class I and II antigens play an important role in demyelination following infection by MHV of resident cells of the CNS. Infection of the CNS with MHV-A59, a neurotropic murine coronavirus, induces class I MHC antigen expression on oligodendrocytes and astrocytes (Suzumura et al. 1986; Suzumura et al. 1988; Lavi et al. 1989), cells that do not normally express detectable MHC surface antigens. The role of MHC class I in demyelination remains complex because of reports indicating that demyelination can occur in MHV-infected mice that lack either stable 
expression of the MHC class I molecule or functional CD8+ T cells (Gombold et al. 1995). Because MHC class I antigens play a key role in interaction between cytotoxic T cells and target cells, the induction of MHC class I antigens could potentially allow glial cells to interact with or become a target for immunocytes.

The MHC class II molecules play a critical role in induction of immune responses through presentation of processed antigens to $\mathrm{CD} 4^{+} \mathrm{T}$-helper cells. Although class II MHC molecules are essential for lymphocyte development, antigen presentation, and T-cell activation, inappropriate class II expression has been implicated in several autoimmune diseases, including rheumatoid arthritis, inflammatory bowel disease, and MS (Grusby and Glimcher 1995). Class II MHC molecules are normally expressed on professional APCs, such as $\mathrm{B}$ cells, macrophages, dendritic cells, and thymic epithelium, expression on other cell types, including astrocytes, can be induced and/or regulated by cytokines, neurotransmitters, and neuropeptides. Astrocytes were the first CNS cell type shown to express class II MHC molecules upon IFN- $\gamma$ stimulation in vitro (Wong et al. 1984). The cytokine TNF- $\alpha$, while having no influence alone on class II MHC expression, enhances IFN- $\gamma$-induced class II MHC expression on astrocytes (Panek et al. 1994). Infection of astrocyte cultures derived from MHV-susceptible Lewis rats with MHVJHM resulted in induction of MHC class II, whereas no such induction was observed in astrocytes from disease-resistant Brown Norway rats (Massa et al. 1986; Massa et al. 1986). A number of soluble mediators have been shown to inhibit class II MHC expression in astrocytes, which include TGF$\beta, \quad I L-1 \beta, \quad$ IFN- $\beta, \quad I L-4, \quad$ glutamate, vasoactive intestinal peptide, norepinephrine, and nitric oxide (Rohn et al. 1996). The expression of MHC II on phagocytic microglia implies the capacity of microglia as APCs. Phagocytic microglia is often regarded as potentially cytotoxic in both acute and chronic CNS disorders. MHC class II expression by phagocytic microglia also corresponds to the elevated expression of potentially cytotoxic substances in the CNS, among which nitric oxide and TNF- $\alpha$ are notably involved in the death of oligodendrocytes and demyelination (Benveniste 1997; Eugster et al. 1999). A study on the demyelinating twitcher mouse has shown that the number of reactive microglia and the concomitant demyelination is significantly reduced when the twitcher mouse carries an MHC II null background (Matsushima et al. 1994). Therefore, MHC II expression by activated microglia in chronic neurodegenerative disorders may indicate that the activated microglia become cytotoxic (Zhang et al. 2001).

Expression of MHC class II has been shown to be important in demyelinating disease in some animal models, such as TMEV and EAE (Borrow and Nash 1992). However, mice deficient in MHC class II 
expression are capable of having demyelination following infection with MHV-JHM (Houtman and Fleming 1996). Therefore, the expression of MHC class I or II may play some role in the MHV-induced demyelination, but neither one is absolutely necessary for this process.

\subsection{Cytokines and chemokines production}

Astrocytes and microglia produce a variety of cytokines and chemokines upon stimulation with a variety of factors including viral infection. Although expression of cytokines and chemokines is controlled and balanced in the normal, healthy, CNS, aberrant expression occurs in CNS diseases such as AD, MS, HAD, Parkinson's disease, and in brain injury/trauma. In many of these diseases, astrocytes and microglia are responsible for some of the production of cytokines and chemokines, whereas blood-borne inflammatory cells are responsible for the rest. Both in vitro and in vivo studies have documented the ability of astrocytes to produce interleukin-1, -6 , and -10 (IL-1, -6 , and -10 ); interferon (IFN)- $\alpha$, and $-\beta$; colony-stimulating factors GM-CSF, M-CSF, and G-CSF; TNF- $\alpha$; TGF- $\beta$; and chemokines including RANTES, IL-8, monocyte chemoattractant protein-1 (MCP-1), and IFN- $\gamma$-inducible protein-10 (IP-10) (Dong and Benveniste 2001). Cytokines that have been demonstrated to be produced by microglia are IL-1, $2,3,4,6,8,10$, IFN- $\gamma$, TNF- $\alpha$, TGF- $\beta$, and CSF. Cytokine/chemokine communications between microglia and astrocytes are involved in the balance of protective and destructive actions by these cells. Both cell types may play a dual role, amplifying the effects of inflammation and mediating cellular damage as well as protecting the CNS. Thus, interactions between T lymphocytes, microglia, and astrocytes play a major role in the pathogenesis of nerological diseases such as MS.

Chemokines are small chemotactic cytokines that modulate leukocyte recruitment and activation during inflammation. Chemokines have been implicated in a variety of normal CNS functions (i.e., neuronal progenitor migration, axon guidance and adhesion, oligodendrocyte proliferation, and intercellular communication) although more and more evidence supports their role in CNS disease and injury such as Alzheimer's disease and HAD. In particular, chemokines are induced in the demyelinating disease MS, and numerous animal models for demyelination including experimental autoimmune encephalomyelitis (EAE), Theiler's and MHV-induced demyelinating disease, experimental autoimmune neuritis, and twitcher, a murine model of globoid cell leukodystrophy (Glabinski and Ransohoff 1999; Hoffman et al. 1999; Kieseier et al. 2000).

Many cells in the body can secrete chemokines, including astrocytes and microglia. MHV infection of the CNS results in an orchestrated expression of chemokine genes including IFN inducible protein of $10 \mathrm{kDa} / \mathrm{CXCL} 10$, 
monokine induced by IFN- $\gamma /$ CXCL9, CCL2, CRG-2, MCP-1, MCP-3, RANTES, Mig, macrophage inflammatory protein-13/CCL4, CCL5, and CCL7 (Lane et al. 1998). Neutralization of IP-10 or Mig at the time of infection with MHV results in increased mortality, higher viral loads and markedly decreased $\mathrm{T}$ cell infiltration into the CNS (Liu et al. 2000; Liu et al. 2001). In contrast, mice treated with RANTES antiserum have delayed viral clearance, decreased $\mathrm{T}$ cell infiltration, and significantly less demyelination than do untreated mice, but show no change in mortality (Lane et al. 2000). Also certain chemokine receptors including CCR1, CCR2 and CCR5, have been shown to regulate the gateway for inflammatory cell entry into the CNS. CCR1-deficient mice have reduced CNS inflammatory responses in the MOG-induced EAE model (Rottman et al. 2000), and CCR5-deficient mice have negligible inflammation after disseminated $C$. neoformans infection of the CNS (Huffnagle et al. 1999). Therefore, chemokines have an important role in host defense as well as demyelination induced by viral infection by attracting $\mathrm{T}$ lymphocytes and macrophages into the CNS.

Cytokines are a large and diverse group of polypeptides ranging in size from 8 to $26 \mathrm{kDa}$, which major function is activation of the immune system and inflammatory responses. In most acute encephalitis cytokines such as IL-1, TNF- $\alpha$, IL- 6 and IFN- $\gamma$ are detected in the CNS of infected mice. Depletion of IFN- $\gamma$ leads to decreased virus clearance and greater mortality. However, neutralization of TNF- $\alpha$ did not appear to affect either $\mathrm{T}$ cell recruitment or virus clearance. Except for astrocytes and microglia, edothelial cells are another possible cellular source of cytokine including $\mathrm{L}$ 1, IL-6 and TNF- $\alpha$, as well as some cytokines receptors IL-1R1 and IL-1R2 .

In our laboratory we analyzed the ability of MHVs with different neurotropic phenotypes to modulate the expression of cytokines in astrocytes and microglia in primary cultures from newborn C57BL/6 mice. The results show that infections of cultures with neurotropic viruses MHV-A59 and JHM up-regulate IL-1 $\beta$, IL-6, IL-12p40, IL-15 and TNF- $\alpha$ mRNA in both astrocytes and microglia, significantly more than infection with the nonneurotropic virus MHV-2. Similar up-regulation was observed in mouse brains during acute encephalitis and in mouse spinal cords during the chronic inflammatory demyelinating disease but a different profile was seen in the liver during acute hepatitis. There are close interactions between the five upregulated cytokines. Studies of MHV-A59 infections in cultures derived from cytokine knockout mice indicate that $\mathrm{IL}-12$ and TNF- $\alpha$ are the upstream molecules that cause the subsequent up-regulation of the other three cytokines. Furthermore we found that infection with neurotropic MHVs induce up-regulation of a set of chemokines in astrocytes and microglia, including MIP-1, MIP-3, MCP-2, MCP-5, MIG. This pattern of 
cytokine/chemokine response in astrocytes and microglia is similar to the Th1 response of lymphocytes and appears to be associated with the neurotropic properties of the virus strain. These studies allow us to study the unique contribution of CNS local immune cells to the process of MHV disease and thus provide new insights into the pathogenesis of MHVinduced neurological diseases as well as the general role of brain immune cells and cytokines in the process of immune-mediated diseases of the CNS (Li et al. 2004).

Several reports studied the role of cytokines in the chronic demyelinating process. The cytokines IL-1 $\beta, \mathrm{TNF}-\alpha, \mathbb{L}-6$ are expressed by astrocytes which are located near the sites of demyelination in chronically infected spinal cords (Perlman 1998). A wide range of cytokines have been detected in MS lesions, and $\mathrm{IL}-1 \alpha$ and $\beta, \mathrm{IL}-6, \mathrm{TNF}-\alpha$, and IFN- $\gamma$ may participate in the CNS demyelination process (Benveniste 1998). Cytokines such as IFN$\gamma$, TGF- $\beta, \mathbb{I L}-1, \mathbb{L}-4$, and $\Pi$ - 10 may regulate class II MHC expression (Han et al. 1999; O'Keefe et al. 1999). Cytokines induce increased expression of chemokines, stimulate adhesion molecules on endothelial cells, and allow leukocyte entry into the CNS. Thus cytokines are involved in the initial phase as well as the development stages of demyelination.

Studies using knockout mice have shown that neither perforin, Fas-FasL, TNF- $\alpha$, IL-10 or IFN- $\gamma$ are required for demyelination (Haring and Perlman 2001). Our own studies in knockout mice indicate that deficiency of IL-6 and IL-12p40 does not decrease the level of demyelination induced by MHV-A59 infection compared to wide type C57BL/6 mice. Thus, none of the single cytokines plays a crucial role in demyelination, however, we still did not rule out that a combination of cytokines may be required for demyelination. Chemokines may play a role in demyelination. RANTES is a proinflammatory chemokine that act as a chemoattractant for a variety of lymphocytic and myeloid cell types including monocytes and granulocytes (Lane et al. 2000). Inhibition of RANTES has been shown to reduce MHV induced demyelination (Lane et al. 1999). An alternative hypothesis is that MHV could potentially damage oligodendrocytes by disrupting the function of astrocytes, which in turn affect oligodendrocytes (Sun and Perlman 1995).

\subsection{Regulation of $\mathrm{Th} 1 / \mathrm{Th} 2$ development}

Activation of $\mathrm{CD}^{+}$T-helper $(\mathrm{Th})$ cells within the $\mathrm{CNS}$ plays an important role in regulating immune responses, inflammation and ultimately repair, during a variety of CNS diseases. The types of Th cells are distinguished by the profiles of cytokines that they produce. Th1 cells produce the cytokines $\mathrm{IL}-2, \mathrm{IL}-12, \mathrm{IFN}-\gamma, \mathrm{LT}-\alpha$, and tumor necrosis factor (TNF), leading to macrophage activation, inflammation, and tissue damage (Rengarajan et al. 2000). Th1 cells have been implicated in the pathogenesis 
of CNS autoimmune diseases such as MS and EAE (Owens et al. 2001). In contrast, Th2 cells produce the cytokines $\mathrm{IL}-3, \mathrm{LL}-4, \mathrm{IL}-5, \mathrm{IL}-6, \mathrm{IL}-7, \mathrm{IL}-10$, IL-11, and IL-13, that mediate humoral immune responses and inhibit macrophage functions (Rengarajan et al. 2000). Within the CNS, Th2 type cytokines play a role in down-regulating Th1 responses and macrophage activation. The presence of $I L-12$ and IFN- $\gamma$ facilitates Th1 development; while IL-4 promotes Th2 differentiation.

Cytokines are the most prominent factors determining the development and polarization of Th cells. However these cytokines are not produced by $\mathrm{T}$ cells but by APCs such as DCs, B lymphocytes and macrophages. Astrocytes and microglia are also the source of cytokines within the CNS that can influences Th1 versus Th2 responses (Aloisi et al. 1997; Stalder et al. 1997). According to our own studies IL-12, the crucial cytokine facilitating Thl development, can be produced by astrocytes/microglia upon MHV infection in vitro. Regulation of class II MHC, B7, and CD40 expression are also involved in the $\mathrm{T}$ cells polarization.

\subsection{NOS activation and NO production}

Nitric oxide (NO) is a pleiotropic molecule with important functions in vascular regulation, neuronal function, and immunological processes. Of the three isoforms of nitric oxide synthase (NOS), NOS2 is recognized as an important inflammatory mediator, with both protective and immunopathological capabilities (Nathan and Xie 1994). Macrophage production of NO by NOS2 is part of the effector phase of the immune response to numerous pathogens, including viruses (MacMicking et al. 1997; Reiss and Komatsu 1998). NOS2-generated NO may contribute to the pathology of demyelination by exerting cytotoxic effects on oligodendrocytes (Merrill et al. 1993) and by regulating proinflammatory factors such as cytokine and chemokine secretion (Remick and Villarete 1996; Brenner et al. 1997; Merrill and Murphy 1997).

Both astrocytes and microglia express NOS-2 and release NO upon virus stimulation in vitro. Moreover, these cells are the likely source of NOS-2 expression during CNS inflammation in vivo (Mitrovic et al. 1996). During the acute phase of MHV infection of mice, expression of NOS2 by macrophages is up-regulated, whereas NOS2 synthesis is confined to astrocytes during the chronic demyelinating disease (Sun et al. 1995; Grzybicki et al. 1997).

The role of $\mathrm{NO}$ and NOS2 in MHV-induced demyelination is still controversial. Inducible NOS2 transiently contributes to MHV-induced demyelination. Inhibition of $\mathrm{NOS} 2 / \mathrm{NO}$ by aminoguanidine (AG), a selective inhibitor of NOS2 activity, slows the progression of MHV-induced demyelination by controlling inflammation and modulating chemokine 
expression in the CNS (Lane et al. 1999). More recent studies show that NOS2 function is not required for demyelination in mice infected with MHV-JHM (Wu et al. 2000). Although NO may have a transient role in the early steps of demyelination, it is not necessary for disease to develop. Infection of NOS2 knockout $(-/)$ and NOS2 $(+/+)$ mice with MHV resulted in similar kinetics of viral clearance from the brain and comparable levels of demyelination. MHV-infected NOS2(-/-) mice displayed a marked decrease in mortality as compared to infected $\operatorname{NOS} 2(+/+)$ mice, which correlated with a significant decrease in the number of apoptotic cells present in the brain. These studies indicate that NOS2-generated NO contributes to apoptosis of neurons but not demyelination following MHV infection (Chen and Lane 2002).

Of note, MHV-induced demyelination differs from that observed in other model systems since inhibition of NO production by AG ameliorates demyelination in rodents with adoptive EAE and mice infected with Theiler's murine encephalomyelitis virus (Cross et al. 1994; Rose et al. 1998).

\subsection{The disruption of the $\mathrm{BBB}$}

The BBB has been assumed to be an effective barrier for blood cells. However, during viral infections or autoimmune diseases of the CNS, T lymphocytes are activated and migrate into the CNS and initiate the cellular events leading to inflammation and demyelination within the CNS white matter. The endothelial BBB has been considered the obvious place of entry of circulating lymphocytes into the CNS. The vascular endothelium is the first element that leukocytes encounter. Immune-specific interactions between lymphocytes and cerebral endothelium have been excluded as an initial mechanism. Adhesion interaction between $\mathrm{T}$ cells and endothelia must precede transmigration (Hart and Fabry 1995). Certain adhesion molecules seem to be critically involved in this process for example intercellular adhesion molecule 1(ICAM-1) and vascular cell adhesion molecule 1(VCAM-1) are up-regulated on the endothelial cells. Dissociated cultures of cerebral endothelial cells prepared from surgical resections of adult CNS tissue express an array of adhesion molecules whose expression is modulated by encounter with $\mathrm{T}$ cells or the cytokines they produce (Calabresi et al. 1997). Chemical mediators, particularly chemokines, regulate the synthesis, surface expression, and avidity of adhesion molecules. The most important adhesion molecule pairs are the selectins $(\mathrm{E}$, $\mathrm{L}$ and $\mathrm{P}$ ), the immunoglobulins ICAM-1 and VCAM-1, and the beta 2 and beta 1 integrins (e.g., LFA-1 and VLA-4), which play a role in a number of pathological processes (Cotran and Mayadas-Norton 1998). Various inflammatory mediators, primarily pro-inflammatory cytokines including 
TNF- $\alpha$ and IL-1 $\beta$, activate endothelial cells. Endothelial cells can also be induced to express MHC class II and B7 in vitro (Prat et al. 2000). Furthermore, they inhibit antigen presentation by other APC in co-cultures. The basis for this inhibition is still unknown.

The BBB may have a protective role against spreading of MHV-A59 into the CNS, by specific restriction of viral entry into endothelial cells of cerebral origin (Godfraind et al. 1997).

\section{CONCLUSIONS}

The effector mechanisms of coronavirus-induced demyelination are complex and largely redundant. The brain resident cells respond to infection by coronavirus by producing various inflammatory mediators including cytokines, chemokines, MHC and NO, which could act in concert to orchestrate an inflammatory pathology in the CNS. This argues for an indirect immunologic mechanism by which coronavirus infection could be implicated in a pathogenesis of the CNS diseases. However, one can conclude from all these data that no single molecule is absolutely required for coronavirus-induced demyelination, neither cytokines, MHC class I or II, nor NOS2. Furthermore, no specific immune cell population is essential for coronavirus-induced demyelination, neither CD4 T cells, CD8 T cells, nor hematogenous macrophages (Gombold et al. 1995; Sutherland et al. 1997). Therefore, coronavirus-induced demyelination may be a result of multiple mechanisms, which still need further investigation.

\section{References}

Abbott, N. J. (2002). "Astrocyte-endothelial interactions and blood-brain barrier permeability." J Anat 200(6): 629-38.

Abbott, N. J. and I. A. Romero (1996). "Transporting therapeutics across the blood-brain barrier." Mol Med Today 2(3): 106-13.

Aloisi, F., G. Penna, et al. (1997). "IL-12 production by central nervous system microglia is inhibited by astrocytes." J Immunol 159(4): 1604-12.

Aloisi, F., F. Ria, et al. (2000). "Regulation of T-cell responses by CNS antigen-presenting cells: different roles for microglia and astrocytes." Immunol Today 21(3): 141-7. 
Aloisi, F., F. Ria, et al. (1998). "Microglia are more efficient than astrocytes in antigen processing and in Th1 but not Th2 cell activation." $\underline{\mathrm{J}}$ Immunol 160(10): 4671-80.

Bauer, H. C. and H. Bauer (2000). "Neural induction of the blood-brain barrier: still an enigma." Cell Mol Neurobiol 20(1): 13-28.

Becher, B., A. Prat, et al. (2000). "Brain-immune connection: immunoregulatory properties of CNS-resident cells." Glia 29(4): 293-304.

Benveniste, E. N. (1997). "Role of macrophages/microglia in multiple sclerosis and experimental allergic encephalomyelitis." $\mathrm{J} \mathrm{Mol} \mathrm{Med}$ 75(3): 165-73.

Benveniste, E. N. (1998). "Cytokine actions in the central nervous system." Cytokine Growth Factor Rev 9(3-4): 259-75.

Borrow, P. and A. A. Nash (1992). "Susceptibility to Theiler's virus-induced demyelinating disease correlates with astrocyte class II induction and antigen presentation." Immunology 76(1): 133-9.

Brenner, T., S. Brocke, et al. (1997). "Inhibition of nitric oxide synthase for treatment of experimental autoimmune encephalomyelitis." J Immunol 158(6): 2940-6.

Calabresi, P. A., L. R. Tranquill, et al. (1997). "Increases in soluble VCAM1 correlate with a decrease in MRI lesions in multiple sclerosis treated with interferon beta-1b." Ann Neurol 41(5): 669-74.

Chen, B. P. and T. E. Lane (2002). "Lack of nitric oxide synthase type 2 (NOS2) results in reduced neuronal apoptosis and mortality following mouse hepatitis virus infection of the central nervous system." J Neurovirol 8(1): 58-63.

Cotran, R. S. and T. Mayadas-Norton (1998). "Endothelial adhesion molecules in health and disease." Pathol Biol (Paris) 46(3): 164-70.

Cross, A. H., T. P. Misko, et al. (1994). "Aminoguanidine, an inhibitor of inducible nitric oxide synthase, ameliorates experimental autoimmune encephalomyelitis in SJL mice." J Clin Invest 93(6): 2684-90.

Dong, Y. and E. N. Benveniste (2001). "Immune function of astrocytes." Glia 36(2): 180-90.

Eugster, H. P., K. Frei, et al. (1999). "Severity of symptoms and demyelination in MOG-induced EAE depends on TNFR1." Eur J Immunol 29(2): 626-32.

Ewing, C. and C. C. Bernard (1998). "Insights into the aetiology and pathogenesis of multiple sclerosis." Immunol Cell Biol 76(1): 47-54

Fierz, W., B. Endler, et al. (1985). "Astrocytes as antigen-presenting cells. I. Induction of Ia antigen expression on astrocytes by $T$ cells via immune interferon and its effect on antigen presentation." $\underline{\mathrm{J}}$ Immunol 134(6): 3785-93.

Fontana, A., P. Erb, et al. (1986). "Astrocytes as antigen-presenting cells. Part II: Unlike H-2K-dependent cytotoxic T cells, H-2Ia-restricted T cells are only stimulated in the presence of interferon-gamma." $\underline{\mathrm{J}}$ Neuroimmunol 12(1): 15-28. 
Gehrmann, J., Y. Matsumoto, et al. (1995). "Microglia: intrinsic immuneffector cell of the brain." Brain Res Brain Res Rev 20(3): 269-87.

Glabinski, A. R. and R. M. Ransohoff (1999). "Chemokines and chemokine receptors in CNS pathology." J Neurovirol 5(1): 3-12.

Godfraind, C., N. Havaux, et al. (1997). "Role of virus receptor-bearing endothelial cells of the blood-brain barrier in preventing the spread of mouse hepatitis virus-A59 into the central nervous system." J Neurovirol 3(6): 428-34.

Gombold, J. L., R. M. Sutherland, et al. (1995). "Mouse hepatitis virus A59induced demyelination can occur in the absence of CD8+ T cells." Microb Pathog 18(3): 211-21.

Grusby, M. J. and L. H. Glimcher (1995). "Immune responses in MHC class II-deficient mice." Annu Rev Immunol 13: 417-35.

Grzybicki, D. M., K. B. Kwack, et al. (1997). "Nitric oxide synthase type II expression by different cell types in MHV-JHM encephalitis suggests distinct roles for nitric oxide in acute versus persistent virus infection." J Neuroimmunol 73(1-2): 15-27.

Han, Y., Z. H. Zhou, et al. (1999). "TNF-alpha suppresses IFN-gammainduced MHC class II expression in HT1080 cells by destabilizing class II trans-activator mRNA." J Immunol 163(3): 1435-40.

Haring, J. and S. Perlman (2001). "Mouse hepatitis virus." Curr Opin Microbiol 4(4): 462-6.

Hart, M. N. and Z. Fabry (1995). "CNS antigen presentation." Trends Neurosci 18(11): 475-81.

Haydon, P. G. (2000). "Neuroglial networks: neurons and glia talk to each other." Curr Biol 10(19): R712-4.

Hoffman, L. M., B. T. Fife, et al. (1999). "Central nervous system chemokine expression during Theiler's virus-induced demyelinating disease." J Neurovirol 5(6): 635-42.

Hoheisel, D., T. Nitz, et al. (1998). "Hydrocortisone reinforces the bloodbrain barrier properties in a serum free cell culture system." Biochem Biophys Res Commun 244(1): 312-6.

Houtman, J. J. and J. O. Fleming (1996). "Dissociation of demyelination and viral clearance in congenitally immunodeficient mice infected with murine coronavirus JHM." J Neurovirol 2(2): 101-10.

Houtman, J. J. and J. O. Fleming (1996). "Pathogenesis of mouse hepatitis virus-induced demyelination." J Neurovirol 2(6): 361-76.

Huffnagle, G. B., L. K. McNeil, et al. (1999). "Cutting edge: Role of C-C chemokine receptor 5 in organ-specific and innate immunity to Cryptococcus neoformans." J Immunol 163(9): 4642-6.

Kieseier, B. C., K. Krivacic, et al. (2000). "Sequential expression of chemokines in experimental autoimmune neuritis." $\mathrm{J}$ Neuroimmunol 110(1-2): 121-9.

Lane, T. E., V. C. Asensio, et al. (1998). "Dynamic regulation of alpha- and beta-chemokine expression in the central nervous system during 
mouse hepatitis virus-induced demyelinating disease." $\underline{\mathrm{J} \text { Immunol }}$ 160(2): 970-8.

Lane, T. E. and M. J. Buchmeier (1997). "Murine coronavirus infection: a paradigm for virus-induced demyelinating disease." Trends Microbiol 5(1): 9-14.

Lane, T. E., H. S. Fox, et al. (1999). "Inhibition of nitric oxide synthase-2 reduces the severity of mouse hepatitis virus-induced demyelination: implications for NOS2/NO regulation of chemokine expression and inflammation." J Neurovirol 5(1): 48-54.

Lane, T. E., M. T. Liu, et al. (2000). "A central role for CD4(+) T cells and RANTES in virus-induced central nervous system inflammation and demyelination." J Virol 74(3): 1415-24.

Lavi, E., D. H. Gilden, et al. (1986). "The organ tropism of mouse hepatitis virus A59 in mice is dependent on dose and route of inoculation." Lab Anim Sci 36(2): 130-5.

Lavi, E., D. H. Gilden, et al. (1984). "Experimental demyelination produced by the A59 strain of mouse hepatitis virus." Neurology 34(5): 597603.

Lavi, E., A. Suzumura, et al. (1989). "Induction of MHC class I antigens on glial cells is dependent on persistent mouse hepatitis virus infection." J Neuroimmunol 22(2): 107-11.

Lee, S. J., T. Zhou, et al. (2000). "Differential regulation and function of Fas expression on glial cells." J Immunol 164(3): 1277-85.

Li, Y., L. Fu, et al. (2004). "Coronavirus neurovirulence correlates with the ability of the virus to induce proinflammatory cytokine signals from astrocytes and microglia." J Virol 78(7): 3398-406.

Ling, E. A. and W. C. Wong (1993). "The origin and nature of ramified and amoeboid microglia: a historical review and current concepts." Glia 7(1): 9-18.

Liu, M. T., D. Armstrong, et al. (2001). "Expression of Mig (monokine induced by interferon-gamma) is important in T lymphocyte recruitment and host defense following viral infection of the central nervous system." J Immunol 166(3): 1790-5.

Liu, M. T., B. P. Chen, et al. (2000). "The T cell chemoattractant IFNinducible protein 10 is essential in host defense against viral-induced neurologic disease." J Immunol 165(5): 2327-30.

MacMicking, J., Q. W. Xie, et al. (1997). "Nitric oxide and macrophage function." Annu Rev Immunol 15: 323-50.

Massa, P. A., R. Dorries, et al. (1986). "Viral particles induce Ia antigen expression on astrocytes." Nature 320: 543-546.

Massa, P. T., H. Wege, et al. (1986). "Analysis of murine hepatitis virus (JHM strain) tropism toward Lewis rat glial cells in vitro. Type I astrocytes and brain macrophages (microglia) as primary glial cell targets." Lab. Invest. 55: 318-327.

Matsushima, G. K., M. Taniike, et al. (1994). "Absence of MHC class II molecules reduces CNS demyelination, microglial/macrophage 
infiltration, and twitching in murine globoid cell leukodystrophy." Cell 78(4): 645-56.

Medzhitov, R. and C. A. Janeway, Jr. (1997). "Innate immunity: impact on the adaptive immune response." Curr Opin Immunol 9(1): 4-9.

Meinl, E., F. Aloisi, et al. (1994). "Multiple sclerosis. Immunomodulatory effects of human astrocytes on T cells." Brain 117 ( Pt 6): 1323-32.

Merrill, J. E., L. J. Ignarro, et al. (1993). "Microglial cell cytotoxicity of oligodendrocytes is mediated through nitric oxide." $\underline{\mathrm{J} \text { Immunol }}$ 151(4): 2132-41.

Merrill, J. E. and S. P. Murphy (1997). "Inflammatory events at the blood brain barrier: regulation of adhesion molecules, cytokines, and chemokines by reactive nitrogen and oxygen species." Brain Behav Immun 11(4): 245-63.

Mi, H., H. Haeberle, et al. (2001). "Induction of astrocyte differentiation by endothelial cells." J Neurosci 21(5): 1538-47.

Minagar, A., P. Shapshak, et al. (2002). "The role of macrophage/microglia and astrocytes in the pathogenesis of three neurologic disorders:

HIV-associated dementia, Alzheimer disease, and multiple sclerosis." J Neurol Sci 202(1-2): 13-23.

Mitrovic, B., J. Parkinson, et al. (1996). "An in Vitro Model of

Oligodendrocyte Destruction by Nitric Oxide and Its Relevance to Multiple Sclerosis." Methods 10(3): 501-13.

Nathan, C. and Q. W. Xie (1994). "Nitric oxide synthases: roles, tolls, and controls." Cell 78(6): 915-8.

Nelson, P. T., L. A. Soma, et al. (2002). "Microglia in diseases of the central nervous system." Ann Med 34(7-8): 491-500.

Nguyen, V. T. and E. N. Benveniste (2000). "Involvement of STAT-1 and ets family members in interferon-gamma induction of CD40 transcription in microglia/macrophages." J Biol Chem 275(31): 23674-84.

O'Keefe, G. M., V. T. Nguyen, et al. (1999). "Class II transactivator and class II MHC gene expression in microglia: modulation by the cytokines TGF-beta, IL-4, IL-13 and IL-10." Eur J Immunol 29(4): 1275-85.

Owens, G. P., M. P. Burgoon, et al. (2001). "The immunoglobulin G heavy chain repertoire in multiple sclerosis plaques is distinct from the heavy chain repertoire in peripheral blood lymphocytes." Clin Immunol 98(2): 258-63.

Panek, R. B., Y. J. Lee, et al. (1994). "Characterization of astrocyte nuclear proteins involved in IFN-gamma- and TNF-alpha-mediated class II MHC gene expression." J Immunol 153(10): 4555-64.

Perlman, S. (1998). "Pathogenesis of coronavirus-induced infections. Review of pathological and immunological aspects." Adv Exp Med Biol 440: 503-13. 
Prat, A., K. Biernacki, et al. (2000). "B7 expression and antigen presentation by human brain endothelial cells: requirement for proinflammatory cytokines." J Neuropathol Exp Neurol 59(2): 129-36.

Reiss, C. S. and T. Komatsu (1998). "Does nitric oxide play a critical role in viral infections?" J Virol 72(6): 4547-51.

Remick, D. G. and L. Villarete (1996). "Regulation of cytokine gene expression by reactive oxygen and reactive nitrogen intermediates." J Leukoc Biol 59(4): 471-5.

Rengarajan, J., S. J. Szabo, et al. (2000). "Transcriptional regulation of Th1/Th2 polarization." Immunol Today 21(10): 479-83.

Rohn, W. M., Y. J. Lee, et al. (1996). "Regulation of class II MHC expression." Crit Rev Immunol 16(3): 311-30.

Rose, J. W., K. E. Hill, et al. (1998). "Nitric oxide synthase inhibitor, aminoguanidine, reduces inflammation and demyelination produced by Theiler's virus infection." J Neuroimmunol 81(1-2): 82-9.

Rottman, J. B., A. J. Slavin, et al. (2000). "Leukocyte recruitment during onset of experimental allergic encephalomyelitis is CCR 1 dependent." Eur J Immunol 30(8): 2372-7.

Sobue, K., N. Yamamoto, et al. (1999). "Induction of blood-brain barrier properties in immortalized bovine brain endothelial cells by astrocytic factors." Neurosci Res 35(2): 155-64.

Sofroniew, M. V., T. G. Bush, et al. (1999). "Genetically-targeted and conditionally-regulated ablation of astroglial cells in the central, enteric and peripheral nervous systems in adult transgenic mice." Brain Res 835(1): 91-5.

Soos, J. M., T. A. Ashley, et al. (1999). "Differential expression of B7 costimulatory molecules by astrocytes correlates with $\mathrm{T}$ cell activation and cytokine production." Int Immunol 11(7): 1169-79.

Stalder, A. K., A. Pagenstecher, et al. (1997). "Lipopolysaccharide-induced IL-12 expression in the central nervous system and cultured astrocytes and microglia." J Immunol 159(3): 1344-51.

Sun, N., D. Grzybicki, et al. (1995). "Activation of astrocytes in the spinal cord of mice chronically infected with a neurotropic coronavirus." Virology 213(2): 482-93.

Sun, N. and S. Perlman (1995). "Spread of a neurotropic coronavirus to spinal cord white matter via neurons and astrocytes." J Virol 69(2): 633-41.

Sutherland, R. M., M. M. Chua, et al. (1997). "CD4+ and CD8+ T cells are not major effectors of mouse hepatitis virus A59-induced demyelinating disease." J Neurovirol 3(3): 225-8.

Suzumura, A., E. Lavi, et al. (1988). "Induction of glial cell MHC antigen expression in neurotropic coronavirus infections. Characterization of the $\mathrm{H}$-2-inducing soluble factor elaborated by infected brain cells." $\underline{\mathrm{J}}$ Immunol 140(6): 2068-72. 
Suzumura, A., E. Lavi, et al. (1986). "Coronavirus infection induces H-2 antigen expression on oligodendrocytes and astrocytes." $\underline{\text { Science }}$ 232(4753): 991-3.

Takiguchi, M. and J. A. Frelinger (1986). "Induction of antigen presentation ability in purified cultures of astroglia by interferon-gamma." $\underline{\mathrm{J} \mathrm{Mol}}$ Cell Immunol 2(5): 269-80.

Tran, N. D., J. Correale, et al. (1999). "Transforming growth factor-beta mediates astrocyte-specific regulation of brain endothelial anticoagulant factors." Stroke 30(8): 1671-8.

Utsumi, H., H. Chiba, et al. (2000). "Expression of GFRalpha-1, receptor for GDNF, in rat brain capillary during postnatal development of the BBB." Am J Physiol Cell Physiol 279(2): C361-8.

Weber, F., E. Meinl, et al. (1994). "Human astrocytes are only partially competent antigen presenting cells. Possible implications for lesion development in multiple sclerosis." Brain 117 (Pt 1): 59-69.

Williams, K., E. Ulvestad, et al. (1994). "Immune regulatory and effector properties of human adult microglia studies in vitro and in situ." $\underline{\mathrm{Adv}}$ Neuroimmunol 4(3): 273-81.

Wolburg H, R. W. (1995). Formation of the blood-brain barrier. Neuroglia. K. H. Ransom BR New York, Oxford University Press: 763-776.

Wong, G. H., P. F. Bartlett, et al. (1984). "Inducible expression of H-2 and Ia antigens on brain cells." Nature 310(5979): 688-91.

Wu, G. F., L. Pewe, et al. (2000). "Coronavirus-induced demyelination occurs in the absence of inducible nitric oxide synthase." J Virol 74(16): 7683-6.

Zhang, S. C., B. D. Goetz, et al. (2001). "Reactive microglia in dysmyelination and demyelination." Glia 34(2): 101-9. 